\title{
LËGISLATION
}

\section{Les Droits d'Enregistrement des Concessions de Distribution d'Énergie à l'origine, au moment des cessions, et au sujet du rachat.}

\author{
(SUITE ET FIN)
}

Par Paul BOUgault, Auocal à la Cour d’Appel de Lyon.

TROISIENE PARTIE

\section{Puchat de la Concession par la Commene}

Cetle hypothèse est plus compliquée, comme toutes celles dans lesquelles il n'existe pas de terminologie très claire. I.e mot "rachat " est évidemment bizarre quand on l'applique à la matière des concessions. Pour "racheter ", il faut avoir été propriétaire, avoir vendu et reprendre des mains de l'acquéreur ce qu'on lui a transmis : le pacte de réméré, prévu par le Code Civil et assez fréquent dans la pratique, est le droit que le vendeur se réserve pour le cas où il aurait des regrets, d'annuler son acte et de "redevenir ". propriétaire. Nous disons "redevenir" parce que, dès la première vente, il a certainement cessé de l'être.

Au contraire, quand une commune donne à un concessiommaire le droit d'établir une distribution électrique et, par conséquent, de placer des ouvrages dans son sol public, elle n'entre pas en possession même de ces ouvrages : donc, elle ne peut pas les céder à son concessionnaire, et - c'est l'évidence même - ne les ayant ni possédès ni cédés, comment les rachèterait-elle ?

En réalité, ayant donné au concessionnaire un droit d'exploiLation, elle le prend avant le temps fixé et elle indemnise ledit concessionnaire du préjudice que lui cause une aussi brusque décision dont il est victime. Donc, elle ne "rachìte" rien. Pour expliquer ce mot, on ne trouve qu'un moyen : c'est de décomposer l'opération, au moment où elle s'accomplit, et de supposer qu'à la minute même où se signe l'acte, la commune pendant un instant de raison, s'est considérée comme en droit d'exploiter, qu'elle a immédiatement cédé ce droit à un tiers pour un temps donné, et qu'ensuite, avant l'expiration normale, elle reprend lexploitation et verse une indemnité sous forme d'annuités. Gràce à ce raisonnement subtile et tendancieux jusqu'à l'invraisemblance, le Trésor peut se croire en droit d'appliçuer un tarif à l'indemnité ; c'est véritablement excessif. Mais puisque, comme nous alions le dire, la Cour de Cassation a consacré la thèse d'un paiement fiscal, il faut envisager sérieusement la question.

\section{Et d'abord, quel sera le larif?}

La Cour admet qu'en réalité, la Ville rachìte le droit d'exploiter tlle-même, et cette valeur de l'exploitatiou est considérée comme un droit mobilier justiciable autrefois du tarif de $2 \%$ et aujourd'hui du tarif de $5 \%$; car l'article 24 de la loi du 25 juin 1920 a augmenté le tarif qui était primitivement fixé par l'article 69, paragraphe 5, No 1 de la loi de Frimaire an VII.

Parmi les arrêts qui ont consacré celle thèse, on peut citer celui de la Chambre Civile du 17 juillet 1905 (Dal. 1908. 1. 34,
Ville de Paris contre Enregistrement) qui a été rendu dans une alfaire où la Ville de Paris avait concédé à des entrepreneurs la construction et la jouissance pour cinquante années de divers marchés contenant des bouliques, et ensuite arait rachelé l'exploitation moyennant une annuité égale à la moyenue des produils nets pendant les trois dernières années. La Régie, ayant estimé qu'il y avait paiement au concessionnaire de l'abandon force de son droit d'exploitation, a perçu $2 \%$ sur le tolal des annuilés cumulées : "cette exploitation, disait-elle, étant une valeur " mobilière du patrimoine de l'entrepreneur "; le Tribunal de Paris avait, au contraire, pensé que la fin d'une exploitation ne saurait être génératrice d'un droit fiscal, caractérisant l'opération de rachat par des termes très heureux : "le concessionnaire " a simplement renoncé aux droits qui lui avaient cté conférés " par le contrat primitif; il n'a rien transmis à la Ville et celle-ci " est revenue à la plénitude de son droit paralysé par la délégation " temporaire qu'elle avait accordée et qu'elle supprime".

Mais nous devons attacher une importance toute particuliere au second arrêt qui relate - et chacun peut en faire son profit les multiples précautions qui avaient été prises - malheureusement inutiles -- ; il a été rendu par la Chambre des Requêtes en date du 19 janvier 1909 (Dal. 1912. 1.353, affaire Ville de Lyon contre Administration de l'Enregistremenl).

Les faits qui ont donné lieu à cet arrêt sont les suivanls : par un contrat administratif du 8 août 1853 , la Ville de Lyon avait concédé à la Compagnie générale des Eaux, pour 99 années, le monopole de la distribution de l'eau dans son périmètre. Ia Compagnie se chargeait d'effectuer les travaux, les achats de terrain, les installations, tout devant revenir gratuitement a la Ville à l'expiration du délai normal. En vertu d'un article formul, la Ville avait le droit de racheter la concession à toute époupue, après les trente premières années, moyennant une annuité payable à la Compagnie, pendant chacune des années restant à courir. Vers la fin d'octobre 1899, la Ville de Lyon signilie à la Compagnie générale des Eaux qu'elle entend user de ce droil de rachat et qu'elle prendra possession du service à partir du $1^{\mathrm{er}}$ janvier suivant; pour être certaine de ne pas être inquiétie au point de vue fiscal, la Compagnie des Eaux fit notilier à la Régle la signification qu'elle venait de recevoir en lui licisanl connaître qu'il lui était dû 58 annuités de $1.319 .854 \mathrm{fr} .50$ t.

Immédiaternent, la Régie réclama à la Ville le $2 \%$ des amnuilés cumulces, c'est-à-dire un paiement de 2 millions, pour lequel clle décerna une contrainte; cette réclamation comprenait également (n'omettons pas de le remarquer) le $2 \%$ sur les approvisionnements restant en magasin à la Compagnie : charbon, conduites, ustensiles, matériel de rechange, ctc...).

La Ville de Lyon, envisageant d'abord les approvisionnemenls 
se refusa à payer à la Régic le $2 \%$ sur la somme qu'ils représculaient, n'offrant que le $1 \%$; elle se basait sur l'article $51, N^{\circ} 3$ de la loi du 28 avril 1816 qui vise les marchés " pour constructions, "réparations, entretien, approvisionnements et fournitures "dont le prix doit être payé par les Administrations locales". Iille exejpait de ces mots très généraux" " Lournitures, approvisionnements" ne comportant en eux-mèmes le germe d'aucune distinction ; d'autre part, il était bien évident qu'elle payait elle-même à titre d'administration locale ce qu'elle trouvait en magasin.

L'Avocat général à la Ciour de Cassation, M. Feuilloley, fut le cet avis, mais, pas plus que la Ville de Lyon, il n'arriva a le faire triompher : la Cour supı ême a attaché un sens très strict au mot "marché de fournitures" emplové par l'article 51, No3 de la loi de 1816 , le tarif de $1 \%$ est réservé à ce contrat spécial yui prépare une transmission de propriété sur une chose, sans cxculer cotte transmission immédiatement ; qu'elle soit pascée de sure à gré, ou qu'elle soit passée par adjudication, une convenfion faite pour exiger postérieurement une livraison, mérite seule le mot de " marché ". Quand une ville trouve à pied d'œurre une chose qu'elle est contrainte de prendre en vertu d'une concession, elle réalise seulement un achat ordinaire de choses mobilières qui est justiciable du tarif de $2 \%$ (aujourd'hui $5 \%$ ).

Bestait le point le plus important : était-il dû par la Ville un droit sur les ammuités de rachat? On reprit avec une extraordinaire vigueur, devant la Cour suprême, les arguments de défense précilćs. La Chambre des Requêtes a admis le même système que la Chambre cirile dans le précédent arrêt : l'exploitation est devenue cntre les mains du concessionnaire, une valeur mobilière ; mais la Cour complète l'idée en disant que le fait pour la Ville de mendre celte exploitation pour elle-même constitue une "rétrocession "; on appelle ainsi le fait juridique qui, sans affccler le passé, sans être une annulation rétroactive, ramène pour l'avenir, un bien dans le patrimoine de celui qui l'a transmis. Or, à part les cas énumérés par des textes spéciaux, dans lesquels unc rétrocession n'est soumise qu'au droit fixe, elle donne lieu au même droit qu'une cession ordinaire. (Voir sur ce point Wall, Tome I, No 268, 276.)

1)one, les villes çui rachèteront devront payer les frais de $5 \%$

Jusqu'à présent, nous n'avons examiné que des affaires dont le mchal aboutit au paiement d'une indemnité sous forme d'annuités. Quelle sera la dicision, en présence de l'arlicle 23 du cahicr-type qui, comme on le sait, comprend deux sortes de paicment? La première est mixte : l'annuité se complète par la prestation d'un capital représentant la valeur des frais de premier établissement non amortis ; la scconde ne comporte pas d'annuité ; c'est te remboursement du capital de premier établissement, les frais de constitution de Société et les insuffisances de receltes pendant sept ans au maximum, les insuffisances étant définies par lit différence entre les receltes brutes ct les charges du capital (l'intérêt nornal élant celui de la rente française la plus avantageuse, augmente de deux points).

Que le lise prenne le tarif de $5 \%$ (autrefois $2 \%$ ), c'est l'applicalion de la jurisprudence ci-dessus analysée; le fisc poussera-t-il l'exigence jusqu à ajonter au cumul des annuilés, le capilal représentant le remboursement du premier établissenent ? Il convient d'avoir sur ce point un scepticisme absolu, tant est grande l'extension des appétits fiscaux. Ce serait, en tout cas, une prétention que nous ne saurions comprendre.

En ce qui concerne les annuités, on peut encore s'expliquer je ne dis pas approuver - que, pour percevoir un droit, l'Administration ait créé la théorie suivante : "la commune a aperçu " une cxploitation en bénéfices ; elle la prend pour que ces "bénéfices, à l'avenir, Jui appartiennent. Les annuités seront " considérées comme la simple rancon de ce bénéfjce futur; " donc, je percois, sur ledit prix, le tarif ordinaire des cessions " mobilières. "

Mais le remboursement du capital de premier établissement, dans sa partie non amortie, n'est pas et ne peut pas être le prix d'une cession ou l'une rétrocession : c'est simplement la compensation d'un préjudice, plus exactement, c'est le versement d'une somme destinée à empêcher qu'une injustice se produise : le concessionnaire a fait tous les frais des travaux; il avait toute la durée de la concession pour se rembourser du montant; lui supprimer cette possibilité, c'est rendre obligatoire pour la commune la restitution d'une avance ; ce n'est pas acquitter pour la commune le prix d'une cession d'exploitation en bénéfice.

Le même raisonnement s'applique à plus forte raison, à la seconde méthode de rachat qui consiste dans la restitution du capital de premier établissement et le paiement des insuffisances : il serait vraiment étrange que l'on vînt dire, en présence d'unc exploitation qui ne donne pas de bénéfice, que la commune "rachète ", pour le faire sien, "l'avantage " d'y procéder elle-même.

\section{QUATRIEME PARTIE}

Hypothlise mixte : Les anciennes Concessions et le Rachat des Installations en Fin de Concession

Sous ce litre, nous devons étudier une sorte de contrat qui se présentait très fréquemment avant le cahier des charges-type et était caractérisée par la combinaison suivante : sans attribuer à la commune le droit de prendre gratuitement à l'expiration du délai fixé, les ouvrages et le matériel, la concession lui donne celui de les réclamer moyennant le prix à déterminer par une expertise ; le libellé de la clause affecte en général cette forme : "à l'expiration de la concession, la commune de X... aura le droit " de racheter à sa convenance tout ou partie du matériel d'exploi"tation comprenant les immeubles, le réseau téléphonique, " les appareils et les machines de toute nature, la ligne télégra" phique. La valeur en sera fixée par expertise. Dans le cas où la " commune ne se prévaudrait pas de cette faculté, le concession" naire sera tenu d'enlever à ses frais tout le matériel d'exploita"tion lui appartenant, situé sur ou sous la voie publique et de "remettre les lieux en leur état primitif, etc..."

Dans ces conditions, quel sera le droit de l'Administration?

11 est évident que si elle a déjà perçu, à la première présentation de l'acte, un droit de $1,25 \%$ sur l'évaluation des travaux susceptibles de revenir à la Ville, elle ne peut recevoir qu un supplément calculé sur la différence entre la valeur définitive des ouvrages qui seront remis à la Ville et celle de l'estimation primitive.

Mais nous sommes convañncu que, même dans le cas oùr, pour une raison quelconque, le fisc n'aurait pas, dès la première présentation, réclamé $1,25 \%$ sur l'évaluation, ce tarif serait seul applicable i l'exclusion de tout autre, au montant total des travaux reutrant dans le patrimoine communal.

En effet, dès le début, le contrat a èté rédigé sous l'influence d'une condition suspensive; celle -ci ne jouera qu'à l'expiration du délai et l'on peut dire que les ourrages, au fur et à mesure qu'ils sont établis, sont placés pour le compte de qui il appartiendra.

A la fin de la concession, deux hypothèses peuvent se présenter : ou bien, la Wille exigera l'enlèvement, ct, dans ce cas, les 
ouvrages n'ayanl jamais été compris dans le marché, ne devraient donner lieu à ancune perception, car ils constituent une simple occupation de voirie qui fait partie integrante de loule concession de distribution: ou bien, la Ville use de son droit d'acquisition et alors les ouvrages apparaissent comme ayant été établis pour son compte dès le début, et le tarif de 1,25\% qui aurait été exigé si à l'origine le principe avait été définitif, ne doit pas varier.

Dans ce qui précède, nous n'avons envisagé que les ourrages placés sur le territoire public, et notamment sur celui appartenant à l'autorité concédante, ou mème dans des immeubles achetés par le concessionnaire, soit à l'amiable, soit, au besoin, par expropriation.

Dans les deux cas, en effet, les acquisitions immobilières ont été faites pour le compte de la Ville et dans la remise qui est opérée, on ne doit tenir compte que des ouvrages établis. Il n'y aurait qu'une exception d'ailleurs très spéciale : c'est le cas où le concessionnaire remettrait à l'autorité concédanle des terrains lui ayant appartenu, et à lui seul, sans aucune intervention de ladile autorité. Le droit immobilier de cession pourrait alors être perçu (voir Dictionnaire des Rédacteurs, Vo Marchés No269), et il y aurait lieu de donner dans l'acte même la distinction entre les valeurs immobilières et les aulres valeurs, pour ne pas ètre exposé à payer sur le tont, le droit le plus fort : car, si l'on se rapporte à l'article 9 de la loi de Frimaire an VII, on troure cette règle bien connue: "lorsqu'un acte translatif de propriété * ou d'usufruit comprend les meubles et les immeubles, le droit " d'enregistrement est perçu sur la totalité du prix, à moins qu'il " ne soit stipulé un prix particulier pour les objets mobiliers et " qu'ils ne soient désignés et estimés, article par article, dans le "contrat $"$.

C'est d'ailleurs un immeuble de cette nature (usine à gaz) qui a fait l'objet d'un arrêt extrêmement important de la Chambre des Requêtes du 11 novembre 1918 (Ville de Paris contre Administration de l'Enregistrement, voir mon article sur ledit arrêt paru dans le Journol L'Industrie Electrique du 10 mars 1919). Le 3 novembre 1905, la concession de la Compagnie Parisienne du Gaz était arrivée à expiration, et le 12 janvier 1906 un traité intervenait entre les deux parties contractantes, pour régler les conditions de la remise à la Ville de Paris de la totalité de l'actif de l'établissement de la Compagnie concessionnaire. Ce traité reconnaissait la Ville propriétaire de la moitié de l'actif, depuis une date déjà ancienne et, pour mettre fin à cette indivision, les liquidateurs de la Compagnie ont donné, par le moyen d'une licitation à l'amiable, à la Ville toute la part qui appartenait à la Compagnie, part estimée à 90 millions. L'Administration fiscale affecta, de croire que la Ville de Paris n'avait jamais été propriétaire de la moitié et qu'en réalité elle recevait, pour la première fois, tout un actif valant par conséquent 180 millions et lui demanda le droit de vente au taril de $7 \%$, exigeant le paiement de 12 millions en chiffes ronds. Ia Ville protesta et dit : " je n'acquiers pas la totaliké de cet actil, puisque j'élais "déjà propriétaire d'une part el je ne dois que le taux de $5 \%$ " sur la moitie de l'actil qui mest transmise, soit 4.500 .000 lr.; "c'est donc 8 millions qui m’ont été demandés en trop". kin effet, quand une personne est déjà proprićtaire d'un immeuble pour partic, el quand elle acquiert l'autre partic, le droit primitif qui élail de $4 \%$ en rerlu de l'article 69 , paragraphe $7 \mathrm{~N}^{\circ} 4$ de la loi du 22 Frimaire an VII n'a été modifié et porté à $8 \%$ (que par l'article $25,3^{\mathrm{e}}$ paragraphe de la loi du 25 juin 1920 , done bien après l'arrêt précité.

Par conséquent, la Ville de Paris était en droit dabord de prétendre à un tarif réduit el ensuite de ne vouloir payer que sur la moitié qu'clle acquérait réellement. C'est pourquoi nous avons assisté à des efforts considérables faits par l'Enregistremenl, d'une part, pour démontrer que la Ville de Paris n'avait jamais eu la moindre propriété, et, par celle-ci, d'autre part, pour revendiquer un droit de cetle nalure, en le faisant remonter à une époque aussi lointaine que possible. Le Tribunal a donné raison à la Ville de Paris qui justifiait que, le 23 juillet 1855 , le premier traité qui donnait pour 50 ans la concession à la Compagnie, stipulait que le 31 décembre 1905 , date de l'expiralion, la ville deviendrait, de plein droit, propriétaire des canalisations placées sous les voies publiques sans indemnité, et des usines, moyennant un prix à fixer à dire d'expert. Mais deux autres traités avaient eu lien, du 25 jamier 1801 et 7 février 1870 , au moment de l'annexion à la capitale de certaines communes suburbaines. Or, dans ces deux traités, il élait dit qu'à la fin de la concession " le produit de l'actif mobilier et immobilier de la Compagnie "ferait partie des bénéfices à partager entre les deux parties " contractentes". C'est en se basant sur celte co-propriélé que le Tribunal et, après lui la Cour de Cassation, a dèclaré que le tarif réduit était seul exigible, et encore seulement sur la partic nouvellement acquise. (I'arrêt précité a élé rendu sur le rapport de M. le Conseiller Feuilloley, les conclusions de M. l'Avocat général Blondel, et la plaidoirie de Me de Ségogne, substituant Me Coche, mobilisé, et Me Aubert.)

On ne sera point étonné que nous ayons domé à cette question d'enregistrement des concessions une place aussi considérable; ainsi qu'on vient de le voir, c'est à l'origine mème de la concession que la question se pose et elle se produit encore pendant le cours de la durée, et nème à la fin, pour cerlaines conventions très spéciales.

Nous espérons que, dans les détails ci-dessus, ceux qui auront maille à partir avec le fisc pourront trouver des renseignements susceptibles de les intéresser et au besoin de les défendre. 